Baltic Astronomy, vol.23, 123-135, 2014

\title{
A TALE ON TWO CLOSE BINARIES IN PEGASUS
}

\author{
H. V. Şenavc1 ${ }^{1}$, M. Yılmaz ${ }^{1}$, Ö. Baştürk ${ }^{2}$, İ. Özavcı ${ }^{1}$, Ş. Çalışkan ${ }^{1}$, T. Kılıçoğlu ${ }^{1}$ \\ and C. T. Tezcan ${ }^{1}$ \\ 1 Ankara University, Faculty of Science, Department of Astronomy and Space \\ Sciences, TR-06100, Tandoğan, Ankara, Turkey \\ 2 Ankara University Kreiken Observatory, TR-06837, Ahlatllbel, Ankara, Turkey
}

Received: 2014 April 29; accepted: 2014 June 3

\begin{abstract}
We present the simultaneous light and radial velocity curve analysis of two contact binaries in Pegasus using the Wilson-Devinney code. The following absolute astrophysical parameters are determined: masses, radii and effective temperatures. BB Peg is a W-subtype W UMa-type binary, components of which are main sequence stars with $0.50 M_{\odot}$ and $1.40 M_{\odot}$. The radii of its components are $R_{1}=0.81 R_{\odot}$ and $R_{2}=1.28 R_{\odot}$. V407 Peg is an A-subtype contact binary composed of two subgiant components with masses $1.70 M_{\odot}$ and $0.43 M_{\odot}$, and radii $R_{1}=2.17 R_{\odot}$ and $R_{2}=1.25 R_{\odot}$. Comparisons with the theoretical models for solar composition by Girardi et al. (2000) confirms our classification and supports the results.
\end{abstract}

Key words: stars: binaries: eclipsing - stars: fundamental parameters stars: individual (BB Peg, V407 Peg)

\section{INTRODUCTION}

Eclipsing binaries are key objects in determining absolute stellar dimensions (masses, radii, luminosities) with high accuracy, when their light and radial velocity curves are simultaneously analyzed. Stellar structure and evolution models heavily depend on the accuracy of these parameters. With this study, we aim to present the results of our simultaneous Wilson-Devinney (hereafter WD, Wilson \& Devinney 1971) light and radial velocity curve analysis, that we performed in order to derive absolute physical parameters of two eclipsing binaries in Pegasus, namely BB Peg and V407 Peg.

BB Pegasi (HIP 110493, GSC 1682-1542) is a W-subtype W UMa system. Its variability was first noted by Hoffmeister (1931), who found its orbital period to be 4.3 days. In the $1930 \mathrm{~s}$, numerous authors updated its light elements adding new observations (Guthnick \& Prager 1932; Dobronravin 1935; Piotrowski 1936; Nikonov \& Dobronravin 1937). Whitney (1943) was the first who noticed that the orbital period of the star is variable. Since then, the nature of this variation has been discussed in many studies. In most of them, the orbital period was claimed to be increasing due to mass transfer between the components (Cerruti-Sola \& 
Scaltriti 1980; Cerruti-Sola et al. 1981; Awadalla 1988; Qian 2001), while in some others an unseen third body has been proposed which could be the source causing sinusoidal changes and increase of the orbital period (Cetintaş et al. 2004; Kalomeni et al. 2007; Snyder 2008). The light curve analyses by Giuricin et al. (1981) and Leung et al. (1985) showed that BB Peg is a W-subtype contact system. The light level differences between the phases of maxima, and the asymmetries in the profiles of minima have been attributed to surface inhomogeneities due to hot or cool surface spots (Cerruti-Sola et al. 1981; Leung et al. 1985; Çetintaş et al. 2004; Zola et al. 2005; Kalomeni et al. 2007). Hrivnak (1990) published first spectroscopic observations of the system, and determined its first-ever spectroscopic mass-ratio as $0.340( \pm 0.002)$. Lu \& Rucinski (1999) found the mass ratio to be 0.360 from their radial velocity measurements. Finally, Pribulla et al. (2009) determined the spectral type of the system as F7 V but were not able to obtain spectroscopic observations sufficient to compute its orbital elements due to the closure of the David Dunlap Observatory (DDO).

V407 Peg (BD+14 5016, GSC 1720-658, SAO 108714) was found to be variable in the Semi-Automatic Variability Search program at the Piwnice Observatory. Its variability was attributed to a $\mathrm{W}$ UMa-type eclipsing binary by Maciejewski et al. (2002), who also obtained light curves of the system in two different colors $(B$ and $V)$. Maciejewski et al. (2003) determined preliminary radial velocity amplitudes and hence, the mass ratio of the system $(q=0.253 \pm 0.034)$, based on three spectral observations of the system. They performed a WD analysis of their data in mode-3 and found that the primary minimum was a transit indicating Asubtype system. Maciejewski \& Ligeza (2004) later published new radial velocity observations and the spectroscopic orbital parameters of the system. They found the mass ratio to be $0.234 \pm 0.022$ which is close to their preliminary result. The uncertainty of orbital parameters determined by Maciejewski \& Ligeza (2004) was caused mostly by the proximity of some of the radial velocity observations to the conjunctions and partly by a small number of data points (only 13). Rucinski et al. (2008) published new radial velocity observations of the system, this time including 63 data points. They also found the spectral type of the system as F0 V and the mass ratio as $0.256(6)$. Deb \& Singh (2011) solved the light curves of the system obtained during the All Sky Automated Survey (ASAS) with the WD code using the radial velocities provided by Rucinski et al. (2008) and confirmed that it is an A-subtype contact binary with a high contact degree of $81 \%$. Zasche (2011) indicated that both minima profiles were distorted and asymmetric, and the bottom parts of these minima profiles, which were probably total eclipses, are not flat but rather inclined. Recently Lee et al. (2014) published a light curve and a period analysis of V407 Peg. They modeled three-color light curves and the radial velocity observations of both Maciejewski \& Ligeza (2004) and Rucinski et al. (2008) with the WD algorithm. In their model, a hot spot on the secondary component was used to account for the asymmetries and an unseen third light in luminosity.

In this work, we analyze the light curves of these two close binary systems that we observed at the Ankara University Kreiken Observatory (AUKR) and the radial velocity curves obtained by Lu \& Rucinski (1999) and Rucinski et al. (2008). Our analysis relies on the simultaneous solutions of these curves with the WD algorithm and results in absolute parameters of these two contact binaries. We verify the nature of the primary minima, whether they are transits or occultations. In both 
Table 1. Observational properties of variable, comparison, and check stars.

\begin{tabular}{|c|c|c|c|}
\hline & Variable & Comparison & Check \\
\hline & BB Peg & TYC 1682-1525-1 & TYC 1682-1530-1 \\
\hline$\alpha_{2000}$ & $22^{\mathrm{h}} 22^{\mathrm{m}} 56.89^{s}$ & $22^{\mathrm{h}} 23^{\mathrm{m}} 30.39^{\mathrm{s}}$ & $22^{\mathrm{h}} 22^{\mathrm{m}} 48.22^{\mathrm{s}}$ \\
\hline$\delta_{2000}$ & $+16^{\circ} 19^{\prime} 27.83^{\prime \prime}$ & $+16^{\circ} 23^{\prime} 45.68^{\prime \prime}$ & $+16^{\circ} 21^{\prime} 17.03^{\prime \prime}$ \\
\hline Spectral type & $\mathrm{F} 8 \mathrm{~V}$ & - & - \\
\hline$B-V$ & 0.52 & 1.17 & 0.91 \\
\hline \multirow[t]{2}{*}{$V$ mag } & 11.17 & 10.14 & 11.98 \\
\hline & V407 Peg & TYC 1720-986-1 & TYC $1720-880-1$ \\
\hline$\alpha_{2000}$ & $23^{\mathrm{h}} 36^{\mathrm{m}} 55.37^{\mathrm{s}}$ & $23^{\mathrm{h}} 37^{\mathrm{m}} 20.98^{\mathrm{s}}$ & $23^{\mathrm{h}} 36^{\mathrm{m}} 46.21^{\mathrm{s}}$ \\
\hline$\delta_{2000}$ & $+15^{\circ} 48^{\prime} 06.43^{\prime \prime}$ & $+15^{\circ} 47^{\prime} 01.76^{\prime \prime}$ & $+15^{\circ} 50^{\prime} 36.88^{\prime \prime}$ \\
\hline Spectral type & $\mathrm{F} 2$ & - & - \\
\hline$B-V$ & 0.34 & 0.55 & -0.05 \\
\hline$V$ mag & 9.45 & 11.53 & 12.61 \\
\hline
\end{tabular}

systems, we make use of surface spots to account for the observed light curve asymmetries due to solar-type magnetic activity in BB Peg, and a mass transfer between the components in V407 Peg. We located a hot spot on the primary component of the latter system, which is caused by a possible mass transfer from its slightly evolved secondary, a typical feature seen in A-subtype systems. The high overcontact degree (0.74) also supports such a transfer between the evolved companions. We modeled the light curve asymmetries observed in BB Peg with a cool surface spot on its primary component, an assumption often made to account for such asymmetries also known as the O'Connell effect.

\section{OBSERVATIONS AND DATA REDUCTIONS}

All the light curve data presented in this study were obtained with the 40 cm Schmidt-Cassegrain telescope of the Ankara University Kreiken Observatory (AUKR), and an Apogee ALTA U47 CCD camera. Data reduction was performed with the standard packages of IRAF software. ${ }^{1}$ Individual differential magnitudes were computed in the sense the variable minus the comparison star, whose stability was controlled with observations of a check star. Observational properties of these stars, taken from the SIMBAD database and the references therein, are summarized in Table 1. A log of our observations is presented in Table 2. The light elements, used in the computation of the corresponding orbital phases for each variable are listed in Table 3. They were obtained for this study applying the recently published orbital periods and the times of minimum light levels. Radial velocity observations were taken from the paper by Lu \& Rucinski (1999) for BB Peg, and from Rucinski et al. (2008) for V407 Peg in the online data form.

\section{ANALYSIS}

The data of light and radial velocity curves were analyzed with the PHOEBE software, developed by Prs̆a \& Zwitter (2005) as a graphical user interface to the

\footnotetext{
${ }^{1}$ IRAF is distributed by the National Optical Astronomy Observatories, which are operated by the Association of Universities for Research in Astronomy, Inc., under cooperative agreement with the National Science Foundation.
} 
Table 2. Log of the observations.

\begin{tabular}{lccccccc}
\hline System & Obs. dates & \multicolumn{3}{c}{ Numb. of points } & \multicolumn{3}{c}{ Nightly mean errors } \\
& & $B$ & $V$ & $R$ & $\sigma_{B}$ & $\sigma_{V}$ & $\sigma_{R}$ \\
\hline V407 Peg & 2008 Aug 24, 27, Oct 6 & 524 & 549 & 543 & 0.008 & 0.004 & 0.004 \\
BB Peg & 2009 Aug 21, 23 & 512 & 595 & 594 & 0.004 & 0.002 & 0.003 \\
BB Peg & 2009 Oct 2, 4 & 403 & 438 & 440 & 0.005 & 0.004 & 0.003 \\
\hline
\end{tabular}

Table 3. Light elements used in the computation of the orbital phases.

\begin{tabular}{lcc}
\hline System & $\begin{array}{c}\text { Reference epoch } \\
T_{0}(\text { HJD }+2400000)\end{array}$ & $\begin{array}{c}\text { Orbital period } \\
P_{\text {orb }} \text { (days) }\end{array}$ \\
\hline BB Peg & 56212.289435 & 0.361501 \\
V407 Peg & 52558.176792 & 0.636884 \\
\hline
\end{tabular}

WD code. The best agreement between the models and the observations was sought by minimizing the differences $\left(\Sigma(O-C)^{2}\right)$ with the differential correction program integrated in the software and a visual inspection in each run of the program. The orbital inclination $(i)$, the non-dimensional potentials $\left(\Omega_{h, c}\right)$, and the relative monochromatic luminosities $(L)$ were adjusted at the beginning. The temperatures of the primary components $\left(T_{1}\right)$ were fixed to the values obtained from Gray \& Corbally (1994), corresponding to their spectral types determined by Rucinski et al. (2008) and Pribulla et al. (2009) for V407 Peg and BB Peg, respectively. The temperatures of the secondaries $\left(T_{2}\right)$ were adjusted. The limbdarkening coefficients were taken from the van Hamme (1993) tables assuming a logarithmic law. The synchronized rotation around the axes, vertical to the orbital planes in each case, was assumed. After achieving a satisfactory agreement for the geometry of the model, adjusted parameters were also fixed. Then, in the case of light curve asymmetries, e.g. light level differences between the maxima, spot parameters (size, latitude and longitude) were adjusted for better fits to the observed data.

The distances to both systems were calculated by using their apparent magnitudes taken from the Tycho-2 catalog (Høg et al. 2000). The apparent magnitudes were corrected for the interstellar extinction $A_{V}$. We assumed the extinction to reddening ratio to be 3.1 in the computations. Color excesses were obtained from the $100 \mu \mathrm{m}$ dust emissions from the maps of Schlegel et al. (1998). The bolometric corrections were taken from the Flower (1996) tables.

\section{RESULTS}

\subsection{V407 Peg}

The results of our simultaneous light and radial velocity curve analysis for V407 Peg are summarized in Table 4 . We confirm the classification of this system as an A-subtype of W UMa type binaries as suggested by Maciejewski et al. (2003), because the primary minimum is a transit. However, the fact that the total eclipse profiles in the secondary minimum, which were not clearly visible in their light 
Table 4. Results of the simultaneous analysis of the light and radial velocity curves for V407 Peg.

\begin{tabular}{lccc}
\hline Stellar parameter & Hot component & Cool component & Unit \\
\hline Surface temperature $\left(T_{\mathrm{ph}}\right)$ & 6800 & $6446( \pm 60)$ & $\mathrm{K}$ \\
Albedo & 0.5 & 0.5 & - \\
Surface potential $(\Omega)$ & $2.25( \pm 0.03)$ & $2.25( \pm 0.03)$ & $\mathrm{cgs}$ \\
Gravity darkening $(g)$ & 0.32 & 0.32 & - \\
$L /\left(L_{1}+L_{2}\right)[B]$ & $0.80( \pm 0.09)$ & 0.20 & - \\
$L /\left(L_{1}+L_{2}\right)[V]$ & $0.79( \pm 0.05)$ & 0.21 & - \\
$L /\left(L_{1}+L_{2}\right)[R]$ & $0.78( \pm 0.09)$ & 0.22 & - \\
\hline Limb darkening parameter & Hot component & Cool component & \\
\hline $\mathrm{x}[B]$ & 0.795 & 0.798 & - \\
$\mathrm{x}[V]$ & 0.697 & 0.698 & - \\
$\mathrm{x}[R]$ & 0.602 & 0.601 & - \\
$\mathrm{y}[B]$ & 0.258 & 0.256 & - \\
$\mathrm{y}[V]$ & 0.284 & 0.283 & - \\
$\mathrm{y}[R]$ & 0.290 & 0.291 & - \\
\hline System parameter & Value & Error & Unit \\
\hline Mass ratio $(q)$ & 0.256 & 0.006 & - \\
Orbital inclination $(i)$ & 79.8 & 3.0 & $\circ$ \\
Semi-major axis $\left(a_{\text {orb }}\right)$ & 4.01 & 0.04 & {$\left[R_{\odot}\right]$} \\
Distance & 267 & 3 & pc \\
$V_{\gamma}$ & 9.06 & 0.70 & $\mathrm{~km} / \mathrm{s}$ \\
\hline Spot parameter $($ on the hot component $)$ & Value & Error & Unit \\
\hline Temperature factor $\left(T_{\text {spot }} / T\right)$ & 1.08 & 0.02 & - \\
Spot size $\left(\Theta_{\text {spot }}\right)$ & 26.5 & 2.5 & $\circ$ \\
Spot latitude $\left(\Lambda_{\text {spot }}\right)$ & 90 & 5.1 & $\circ$ \\
Spot longitude $\left(\beta_{\text {spot }}\right)$ & 336.5 & 12.5 & $\circ$ \\
\hline
\end{tabular}

Table 5. Absolute physical parameters of V407 Peg obtained by the simultaneous analysis of the light and radial velocity curves.

\begin{tabular}{lccc}
\hline Absolute parameter & Hot component & Cool component & Unit \\
\hline Mass $(M)$ & $1.70( \pm 0.01)$ & $0.43( \pm 0.02)$ & $M_{\odot}$ \\
Radius $(R)$ & $2.17( \pm 0.01)$ & $1.25( \pm 0.02)$ & $R_{\odot}$ \\
Surface gravity $(\log g)$ & $3.99( \pm 0.01)$ & $3.88( \pm 0.01)$ & cgs \\
Bolometric magnitude $\left(M_{\text {bol }}\right)$ & $2.39( \pm 0.02)$ & $3.82( \pm 0.02)$ & mag \\
Luminosities $(L)$ & $8.83( \pm 0.01)$ & $2.37( \pm 0.01)$ & $L_{\odot}$ \\
\hline
\end{tabular}

curves but are evident in ours, are probably caused by the photometric quality difference between the data sets used in each study. This difference resulted in a significant discrepancy in the orbital inclinations (close to $10^{\circ}$ ) found in their and our studies.

We attempted to solve the system's light curve by modeling with addition of 

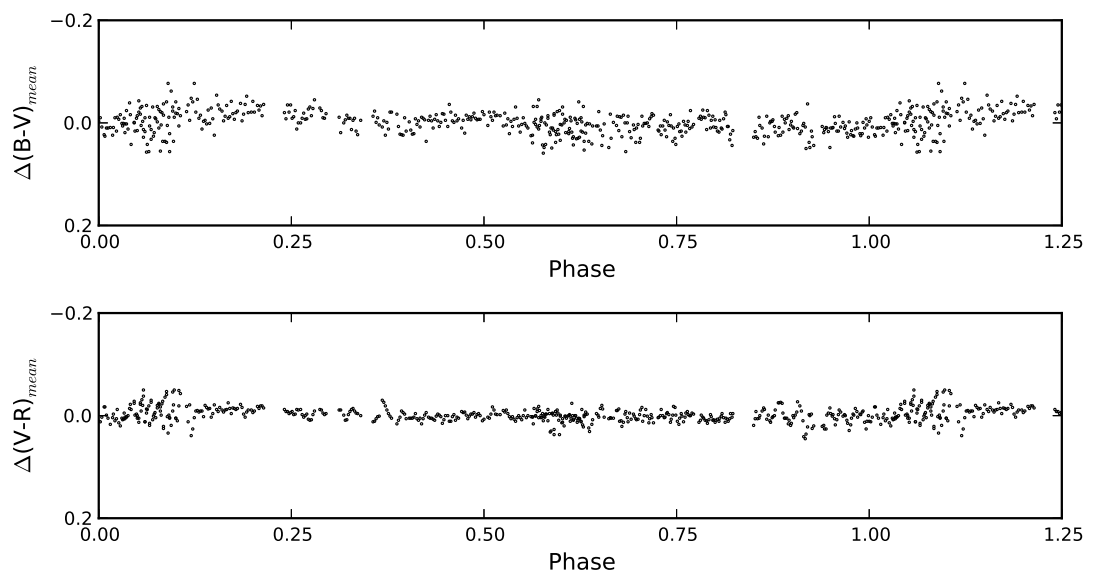

Fig. 1. Color curves $B-V$ and $V-R$ of V407 Peg. The mean color values have been subtracted to better illustrate the variation.

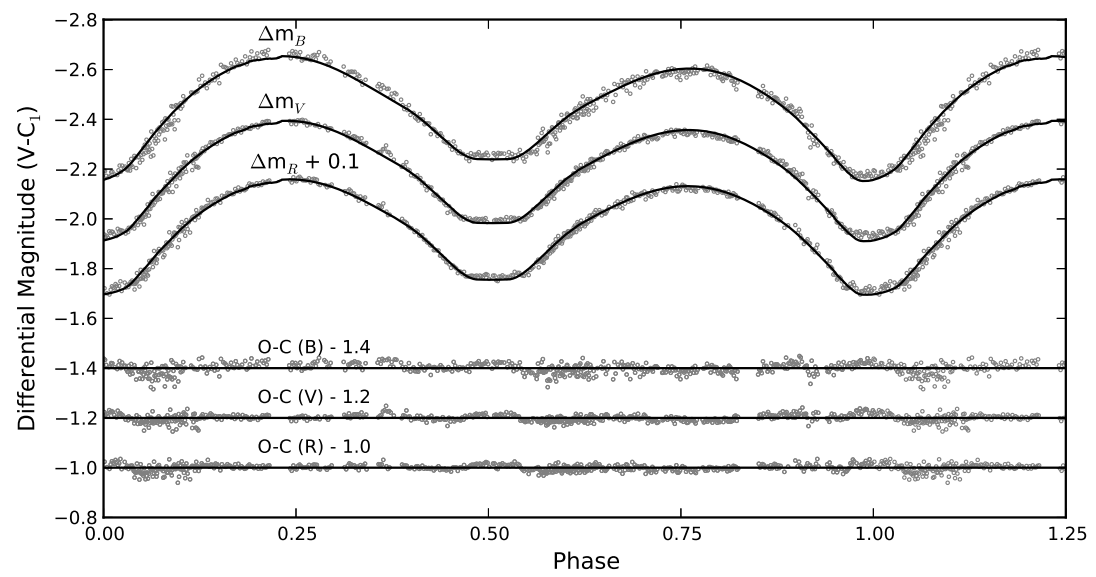

Fig. 2. Observational (circles) and synthetic (solid lines) $B V R$ light curves for V407 Peg. Residuals from the theoretical fits are at the bottom of the figure. Arbitrary shifts for better viewing are indicated in the figure where necessary.

cool and hot surface spots to account for the light curve asymmetries. A hot spot on the primary component with a very large temperature factor (1.55) was the solution of Maciejewski et al. (2003) in modeling the light curve asymmetries. We also modeled the system with a hot spot on the neck region of the binary located on the primary component, with a more realistic temperature factor (1.08) after unsuccessful fits with cool surface spots. The behavior of $B-V$ and $V-R$ colors (Figure 1) after the primary minimum up to the first maximum also supports the hot-spot approach. In an A-subtype W UMa system, some level of reddening is expected in the primary minimum, which contradics the observations showing that the system looks bluer at and around the primary minimum. This behavior is 
indicative of a temperature rise, that can be caused by a hot spot visible around the primary maximum. Zasche (2011) performed a period study of the system using all the times of minima from 2003 until the time of their study. They attributed the observed variations on the $\mathrm{O}-\mathrm{C}$ diagram to possible existence of surface spots, which, they suggest, cause both minima to be distorted and asymmetric. It is too complicated to derive times of minima from such asymmetric profiles, which affect the results of most commonly used methods (e.g. Kwee-van Woerden method, bisector chord method and polynomial fitting) based on symmetric profiles. They do not mention the nature or origin of probable spots, i.e., whether they are magnetic activity induced cool surface spots or hot spots due to mass transfer. In fact, the ASAS light curves, they provided in their study, resemble our light curve in the light level characteristics, the primary maximum being higher than the secondary one by a few hundredths of a magnitude. All the light curves of this system published so far have the same characteristics, which would change in the presence of cool surface spots from one light curve to another due to size and location changes of such spots as it happens in the Sun. Three-color light curves, published recently by Lee et al. (2014), share the same characteristics. The authors of this study also modeled their light curves by attributing the observed asymmetries to a hot spot they placed on the secondary component in their model, which they predicted to originate from a mass exchange between the companions. They speculated that this mass exchange was also responsible for the variation they observed in the eclipse timings. However, the number of timing observations is very limited to reach such a conclusion. We suggest to continue observations for both minima of the light curves in the future and a detailed period analysis, which would tell us whether the observed light curve asymmetries and the proposed $\mathrm{O}-\mathrm{C}$ variations are due to spot(s) caused by mass exchange between the components.

Figure 2 represents the achieved fits to the observations in each of the passbands. The simultaneous light and radial velocity curve solutions gave the absolute parameters presented in Table 5. Rather lower values of surface gravities (3.99 and 3.88) found in our analysis indicate that both components are evolved subgiants. This is supported by a relatively high contact degree (74\%) found in our study.

\section{2. $B B P e g$}

BB Peg was observed with the AUKR T40 telescope in August (8 and 10) and in October (4 and 6) of 2009, twice in each month skipping the night in between to complete two full light curves for each of the months to take into account the orbital phase. We have analyzed these two light curves separately, with the same radial velocity curve obtained by Lu \& Rucinski (1999). The results of our simultaneous light and radial velocity curve analysis for each of these runs are in perfect agreement with each other except for two spot parameters: the temperature factor and the spot longitude.

The BB Pegasi system is a W-subtype W UMa binary according to Binnendijk's classification (Binnendijk 1970), since the primary minimum is an occultation. The contact degree of $18.5 \%$ obtained in this study is also in agreement with this subtype classification. Although there is a considerable amount of scatter in the color curves, some significant degree of the reddening is observed in the primary minimum profiles (Figure 3). Light curves of the system analyzed thus far by many studies have shown that there are out-of-eclipse light level variations due to stellar activity. 
Table 6. Results of the simultaneous analysis of the light and radial velocity curves of BB Peg.

\begin{tabular}{lccc}
\hline Stellar parameter & Hot component & Cool component & Unit \\
\hline Surface temperature $\left(T_{\text {ph }}\right)$ & 6350 & $6163( \pm 45)$ & $\mathrm{K}$ \\
Albedo & 0.5 & 0.5 & - \\
Surface potential $(\Omega)$ & $6.19( \pm 0.02)$ & 6.19 & cgs \\
Gravity darkening $(g)$ & 0.32 & 0.32 & - \\
$L /\left(L_{1}+L_{2}\right)[B]$ & $0.33( \pm 0.05)$ & 0.67 & - \\
$L /\left(L_{1}+L_{2}\right)[V]$ & $0.32( \pm 0.04)$ & 0.68 & - \\
$L /\left(L_{1}+L_{2}\right)[R]$ & $0.31( \pm 0.04)$ & 0.68 & - \\
\hline Limb darkening parameter & Hot component & Cool component & \\
\hline x $[B]$ & 0.810 & 0.820 & - \\
x $[V]$ & 0.717 & 0.733 & - \\
x $[R]$ & 0.624 & 0.640 & - \\
y $[B]$ & 0.227 & 0.200 & - \\
y $[V]$ & 0.274 & 0.263 & - \\
y $[R]$ & 0.281 & 0.273 & - \\
\hline System parameter & Value & Error & Unit \\
\hline Mass ratio $(q)$ & 2.78 & 0.02 & - \\
Orbital inclination $(i)$ & 87.7 & 0.2 & $\circ$ \\
Semi-major axis $\left(a_{\text {orb }}\right)$ & 2.64 & 0.04 & {$\left[R_{\odot}\right]$} \\
Distance & 306 & 8 & pc \\
$V_{\gamma}$ & -28.15 & 0.30 & $\mathrm{~km} / \mathrm{s}$ \\
\hline Spot parameter $($ on the hot component) & Value & Error & Unit \\
\hline 2009 August run & & & \\
Temperature factor $\left(T_{\text {spot }} / T\right)$ & 0.89 & - & - \\
Spot size $\left(\Theta_{\text {spot }}\right)$ & 9.0 & - & $\circ$ \\
Spot latitude $\left(\Lambda_{\text {spot }}\right)$ & 90 & - & $\circ$ \\
Spot longitude $\left(\beta_{\text {spot }}\right)$ & 112.0 & - & $\circ$ \\
\hline 2009 October run & 0.85 & - & $\circ$ \\
Temperature factor $\left(T_{\text {spot }} / T\right)$ & 92 & - & - \\
Spot size $\left(\Theta_{\text {spot }}\right)$ & 272 & & - \\
Spot latitude $\left(\Lambda_{\text {spot }}\right)$ & & & - \\
Spot longitude $\left(\beta_{\text {spot }}\right)$ & $(R)$ & & - \\
\hline & & & - \\
\hline
\end{tabular}

Table 7. Absolute physical parameters for BB Peg obtained by the simultaneous analysis of the light and radial velocity curves.

\begin{tabular}{lccc}
\hline Absolute parameter & Hot component & Cool component & Unit \\
\hline Mass $(M)$ & $0.50( \pm 0.02)$ & $1.40( \pm 0.04)$ & $M_{\odot}$ \\
Radius $(R)$ & $0.81( \pm 0.01)$ & $1.28( \pm 0.02)$ & $R_{\odot}$ \\
Surface gravity $(\log g)$ & $4.32( \pm 0.01)$ & $4.37( \pm 0.01)$ & $\operatorname{cgs}$ \\
Bolometric magnitude $\left(M_{\text {bol }}\right)$ & $4.79( \pm 0.03)$ & $3.97( \pm 0.05)$ & mag \\
Luminosities $(L)$ & $0.94( \pm 0.01)$ & $2.04( \pm 0.01)$ & $L_{\odot}$ \\
\hline
\end{tabular}



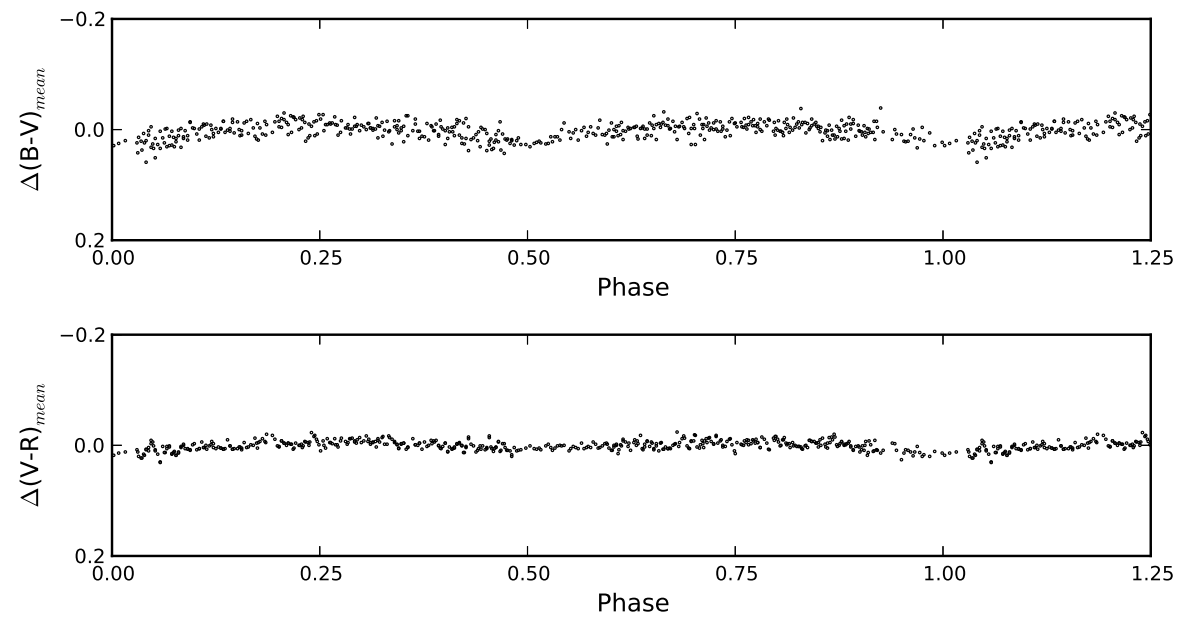

Fig. 3. Color curves $B-V$ and $V-R$ for BB Peg. The mean color values have been subtracted to better illustrate the variation.

Light level difference of the secondary maxima (phase 0.75), being lower compared to the primary maxima (phase 0.25) in both light curves obtained in August and October of 2008, could be attributed to surface inhomogeneities due to solartype spots, a familiar phenomenon in such late spectral type stars. Because, the reddening also stands out more in the secondary maximum, we modeled each light curve with a cool spot positioned to appear in the direction of the observation at this particular phase $(0.75)$ but at longitudes differing by $160^{\circ}$ in the duration of two months. Such a sudden phase jump closely resembles to what is called a flipflop event, defined as a sudden interchange of the more and less active hemispheres on the stellar surface (Korhonen \& Järvinen 2006; Oláh 2010) and observed in some other magnetically active binaries (Jetsu et al. 1991; Berdyugina \& Tuominen 1998; Özdarcan et al. 2012). This abrupt switch of predominant activity region in longitude by $180^{\circ}$ has been observed to repeat itself quasi-periodically (Korhonen et al. 2001). But since we don't have long term photometry of the system evenly spaced in time, we are not in a position to comment on a possible flip-flop cycle for this system. We also tried hot-spot based models, which did not fit the observational data satisfactory.

All our results are summarized in Table 6, while Figures 4 and 5 show the achieved fits to the observations in August and October, respectively. Masses and radii, in particular, agree with those found by Zola et al. (2005).

\section{DISCUSSION}

Our analysis of photometric data together with the existing precise spectroscopic measurements allowed us to estimate the absolute physical stellar parameters of two eclipsing binaries. We confirm the conclusion of Guiricin et al. (1981) and Leung (1985) that BB Peg belongs to the W-subtype and the conclusion of Maciejewski \& Ligeza (2004) that of V407 Peg belongs to the A-subtype. The geometric models we achieved for both these systems gave the best fits to the ob- 


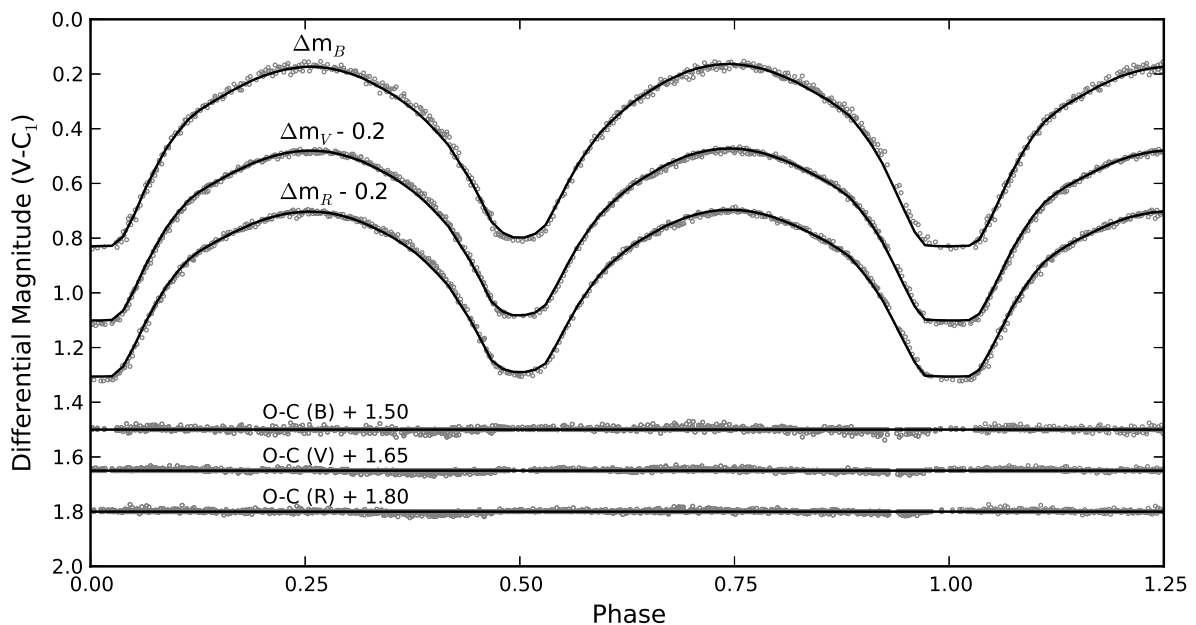

Fig. 4. Observational (circles) and synthetic (solid lines) $B V R$ light curves for BB Peg obtained on 2009 August 8 and 10. The rest is the same as in Figure 2.

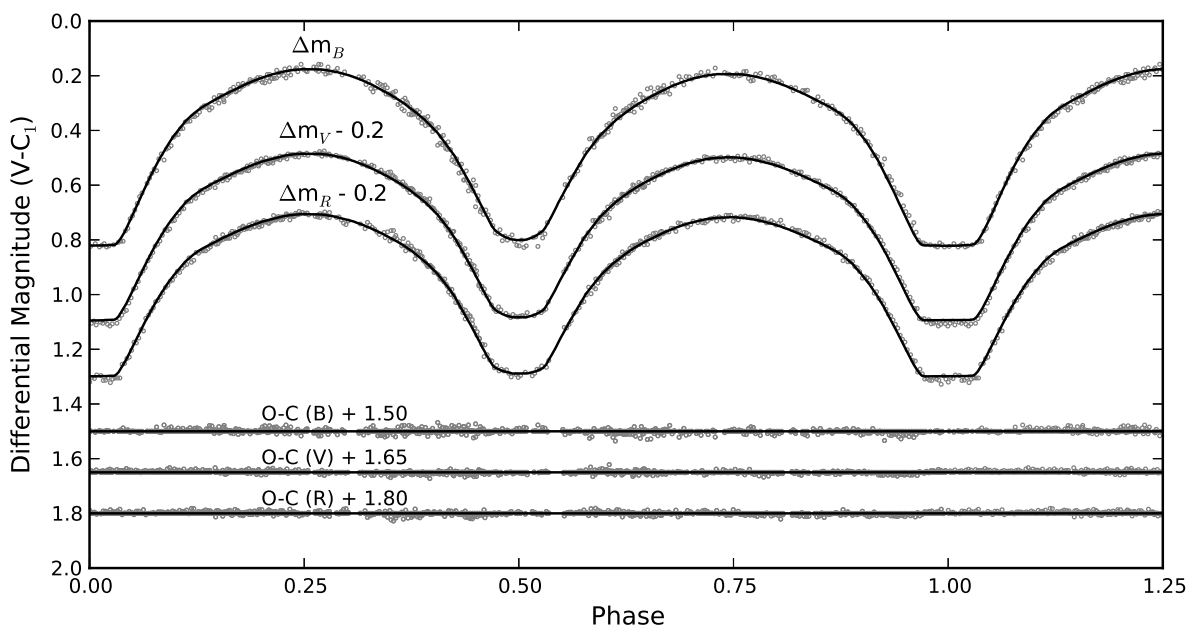

Fig. 5. Observational (filled circles) and synthetic (solid lines) BVR light curves for BB Peg obtained on 2009 October 4 and 6. The rest is the same as in Figure 2.

servations when combined with the surface spot models, which are used to explain the profile asymmetries observed in the light curves. We have modeled the light curves of BB Peg obtained in two different months (August and October of 2008) with the same geometric parameters but different spot parameters changing only in longitude by $160^{\circ}$ and in size, which could be attributed to an active flip-flop mechanism (Berdyugina 2007). We have not detected, within our observation accuracy, a third source of light in the solutions of BB Peg which changes its orbital period as suggested before. The distances we derived for both systems agree within the error limits with those found from the Hipparcos parallax values (van Leeuwen 2007).

The absolute stellar parameters obtained from our models indicate that pri- 

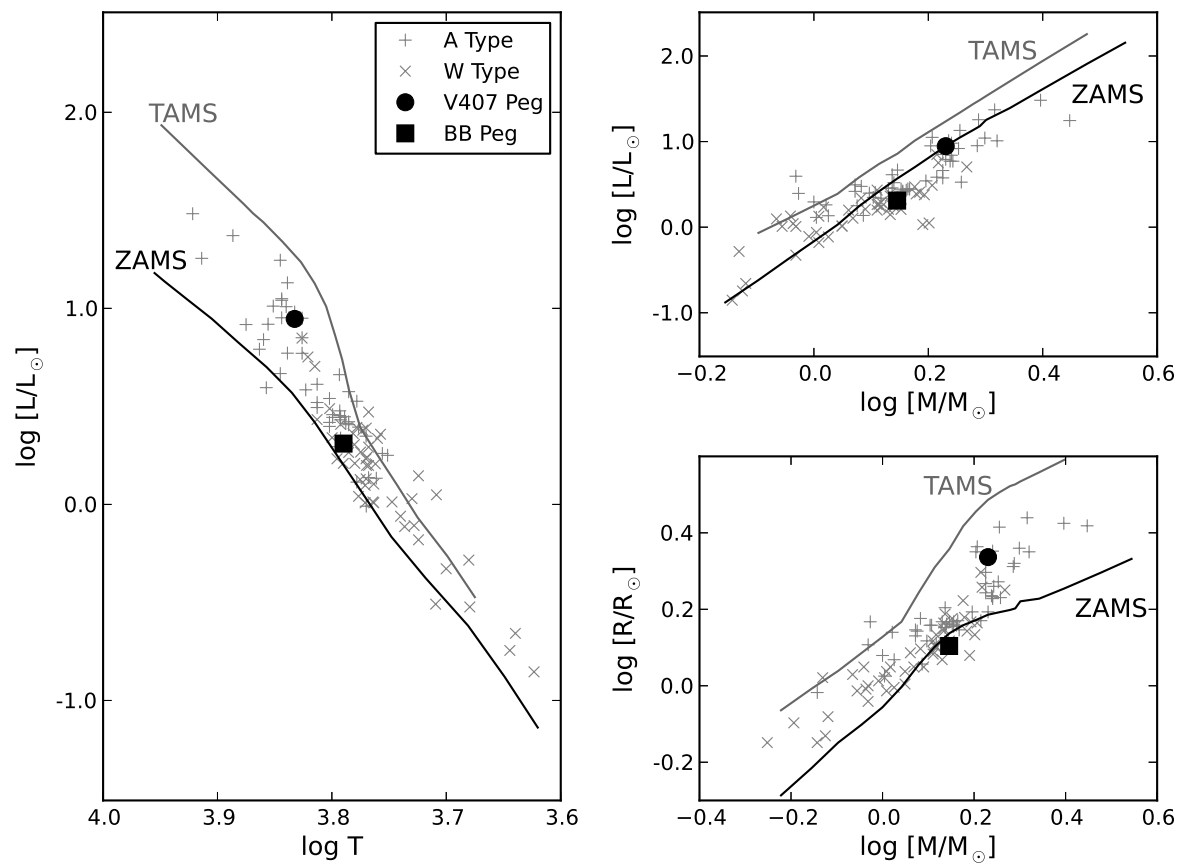

Fig. 6. HRD (left), mass-luminosity (top right), and mass-radius (bottom right) diagrams of the selected set of W UMa primaries, and the primary components of our targets. ZAMS and TAMS data are taken from the theoretical models of Girardi et al. (2000) for solar composition. The legends on the HRD apply to other two diagrams as well.

mary components of both systems lie in the region on the Hertzsprung-Russell diagram (hereafter HRD) between the theoretical zero-age main sequence (ZAMS) and the terminal age main sequence (TAMS) given by Girardi et al. (2000) for solar composition (Figure 6). The evolutionary status of the BB Peg and V407 Peg primaries can be estimated from Figure 6 by comparing their positions with a selected set of A- and W-subtype contact binaries. Their positions of these two systems agree perfectly with their A- and W-subtypes.

The high overcontact degree (0.74) we found for V407 Peg is in agreement with the A-subclass of W UMa stars (Maciejewski \& Ligeza 2004) and the results of previous studies by Deb \& Singh (2011) and Lee et al. (2014). The primary component of this system lies close to the TAMS on the HRD (Figure 6). This position is indicative of a typical A-type primary component that is predominantly more evolved than its W-type counterparts (Lucy \& Wilson 1979). The position of the secondary is also in good agreement with the positions of other slightly evolved A-type system secondaries that have substantially larger luminosities than expected for their ZAMS masses (Yakut et al. 2004). The evolutionary status of this component is also reflected by its relatively small surface gravity $(\log g=$ $3.88)$, and its bolometric brightness $\left(M_{\mathrm{bol}}=3.82 \mathrm{mag}\right)$. This can be the result of mass transfer between the components, from the secondary to the primary in this case, which is supported in our model by a hot spot on the side of the primary 

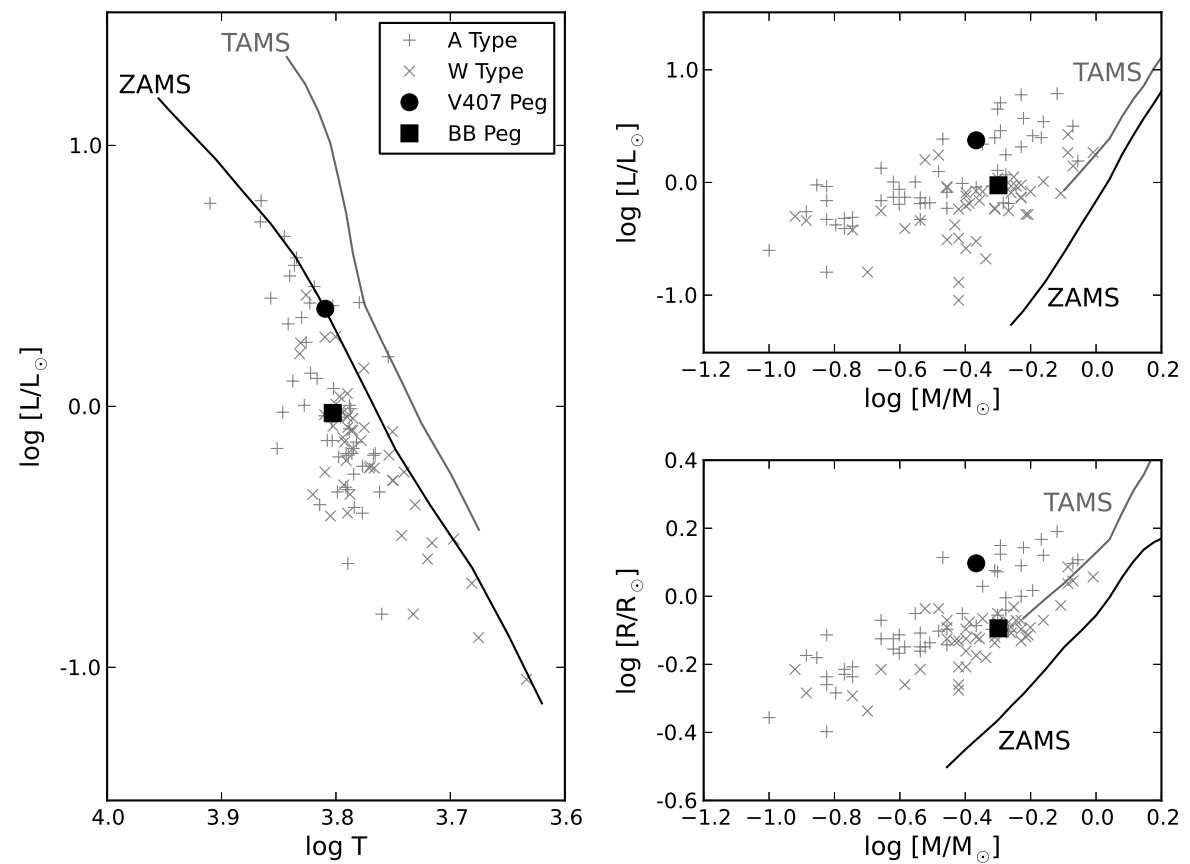

Fig. 7. HRD (left), mass-luminosity (top right) and mass-radius (bottom right) diagrams of selected W UMa secondaries and the secondary components of our targets. The rest is the same as in Figure 7.

facing the secondary. The position of this subgiant secondary component on the ZAMS (Figure 7) is probably due to its evolution in a binary system with mass transfer, rather than as a single star that would evolve differently in the absence of the interaction with a close companion. In fact, the findings of our study and of the recently published paper by Lee et al. (2014) support the mass exchange hypothesis.

ACKNOWLEDGMENTS. We are grateful for the support by the research fund of Ankara University (BAP) through the project 13B4240006. The authors also acknowledge the use of the Simbad and Hipparcos databases. We would like to thank all the staff working for AUKR, Gözde Aydın, Burcu Savran and all the student observers who volunteered in the observations.

\section{REFERENCES}

Awadalla N. S. 1988, Ap\&SS, 140, 137

Berdyugina S. V., Tuominen I. 1998, A\&A, 336, L25

Berdyugina S. V. 2007, Highlights of Astronomy, 14, 275

Binnendijk L. 1970, Vistas in Astronomy, 12, 217

Cerruti-Sola M., Scaltriti F. 1980, A\&A, 40, 85

Cerruti-Sola M., Milano L., Scaltriti F. 1981, A\&A, 101, 273 
Çetintaş C., Şenavcı H. V., Elmaslı A. et al. 2004, XIV Ulusal Astronomi Kongresi, Kayseri, p. 358 (in Turkish, http://80.251.40.59/science.ankara.edu.tr/s elam/pdf_ppt/UAT14_BBPeg.pdf)

Deb S., Singh H. P. 2011, MNRAS, 412, 1787

Dobronravin P. 1935, Variable Stars, Nizhny Novgorod, No. 48

Flower P. J. 1996, ApJ, 469, 355

Girardi L., Bressan A., Bertelli G., Chiosi C. 2000, A\&AS, 141, 371

Giuricin G., Mardirossian F., Mezzetti M. 1981, AN, 302, 285

Gray R. O., Corbally C. J. 1994, AJ, 107, 742

Guthnick P., Prager R. 1932, AN, 247, 121

Hoffmeister C. 1931, AN, 242, 129

Høg E., Fabricius C., Makarov V. V. et al., 2000, A\&A, 355, 27

Hrivnak B. J. 1990, BAAS, 22, 1291

Jetsu L., Pelt J., Tuominen I., Nations H. 1991, in The Sun and Cool Stars: Activity, Magnetism, Dynamos, IAU Colloq. 130, LNP, 380, 381

Kalomeni B., Yakut K., Keskin V. et al. 2007, AJ, 134, 642

Korhonen H., Berdyugina S. V., Strassmeier K. G., Tuominen I. 2001, A\&A, 279, L30

Korhonen H., Järvinen S. P. 2006, IAUS, 240, 453

Lee J. W., Park J.-H, Hong K., Kim S.-L., Lee C.-U. 2014, AJ, 147, 91

Leung K.-C., Zhai D., Zhang Y. 1985, AJ, 90, 515

Lu W., Rucinski S. M. 1999, AJ, 118, 515

Lucy L. B., Wilson R. E. 1979, ApJ, 231, 502

Maciejewski G., Karska A., Niedzielski A. 2002, IBVS, 5343

Maciejewski G., Ligeza P., Karska A. 2003, IBVS, 5400

Maciejewski G., Ligeza P. 2004, IBVS, 5504

Nikonov V. B., Dobronravin P. 1937, Bull. Abastumani Obs., 1, 11

Oláh K. 2010, IAUS, 273, 104

Özdarcan O., Evren S., Henry G. W. 2012, AN, 333, $138 \mathrm{O}$

Piotrowski S. 1936, Acta Astron., 2, 157

Pribulla T., Rucinski S. M., Blake R. M. et al. 2009, AJ, 137, 3655

Prs̆a A., Zwitter T. 2005, ApJ, 628, 426

Qian S. 2001, MNRAS, 328, 635

Rucinski S. M., Pribulla T., Mochnacki S. W. et al. 2008, AJ, 136, 586

Schlegel D. J., Finkbeiner D. P., Davis M. 1998, ApJ, 500, 525

Snyder L. F. 2008, SASS, 27, 153

van Hamme W. 1993, AJ, 106, 2096

van Leeuwen F. 2007, A\&A, 474, 653

Wilson R. E., Devinney E. J. 1971, ApJ, 166, 605

Whitney B. S. 1943, AJ, 50, 131

Yakut K., Kalomeni B., İbanoğlu C. 2004, A\&A, 417, 725

Zasche P. 2011, IBVS, 5991

Zola S., Kreiner J. M., Zakrzewski B. et al. 2005, Acta Astron., 55, 389 